\section{Simultaneous involvement of optic and abducens nerves by Lyme disease: Case report with review of the literature}

\author{
Iman Dabiri, Ahmet Z. Burakgazi \\ Virginia Tech Carilion School of \\ Medicine, Roanoke, VA, USA
}

\begin{abstract}
Ocular manifestations of Lyme disease (LD) remain a rare feature of the disease, but it may present a wide range of clinical presentations with different combinations. LD related optic neuritis or cranial nerve $(\mathrm{CN})$ six palsy have been reported in the literature. However, this is the first case report of simultaneous involvement of CN 2 and CN 6 in a patient with LD. The diagnosis of LD can be challenging and initial laboratory tests can be a false negative. It is paramount important to repeat the diagnostic test if clinical suspicious is ongoing. With this case, we aim to increase awareness of clinicians for possible ocular manifestations of LD and its complex diagnostic process.
\end{abstract}

\section{Introduction}

Lyme disease (LD) is an arthropod-born disease caused by Borrelia burgdorferi and commonly seen in the Northern Hemisphere. Neurological manifestations of LD occur in $12 \%$ of these cases and can present as the only manifestation of the disease as early as 2-18 weeks after infection, and ocular manifestations can be seen in all three stages of the disease. ${ }^{1-6}$ Ocular findings in LD are uncommon, but prior case studies and literature have reported conjunctivitis; keratitis; photophobia; periorbital edema; pupillary abnormalities; cranial nerves $(\mathrm{CN})$ palsies of III, IV, VI; papilledema; optic neuritis; and optic atrophy. $3,6-14$

Optic neuritis $(\mathrm{ON})$ is an inflammation of the optic nerve and is seen in various CNS etiologies, including demyelinating, autoimmune, inflammatory, infectious, and post-vaccination. ${ }^{10,15} \mathrm{ON}$ is usually associated with pain, and the patient usually has a history of rapid visual loss over hours to weeks, an afferent pupillary defect, or optic disc edema, in conjunction with either decreased visual acuity, visual field defect, or dyschromatopsia. ${ }^{15,16} \mathrm{ON}$ in LD is a rare finding, with a handful of reported cases documenting isolated nerve head involve- ment. $15,17,18$ Sixth-nerve innervated lateral rectus muscle and its palsy is hallmarked by double vision that worsens with horizontal gaze in the direction of the paretic lateral rectus muscle and can be an acquired lesion at any point along its path starting from the sixth nucleus located at the dorsal pons. ${ }^{19}$ In adults, etiologies include idiopathic, inflammatory, trauma, tumors, vascular insults, and infectious. ${ }^{20}$ Double vision as a result of palsies to cranial nerves III, IV, and VI have been reported numerous times in various literature associated with LD. ${ }^{12}$ In this case report, we discuss an atypical case of possible LD presenting with ipsilateral left $\mathrm{ON}$ and left sixth-nerve palsy, along with pre- and post-treatment findings and literature review.

\section{Case Report}

A 56-year-old female with past medical history of migraine headache and fibromyalgia presented on October 24, 2018 with continuous onset of left-sided hemifacial pain/headache for one week prior to the development of diplopia and blurry vision in her left eye. She claimed to have had tick exposure in June of 2018. The patient initially expressed a sudden and severe onset of a left temporal headache. The pain was described as sharp knives being stuck into her left temple/head and face extending to her nostril. This was associated with nausea without vomiting, which was atypical for her normal migraine attacks. She tried sumatriptan, but it was not effective. She experienced subjective changes in her taste and hyperalgesia to the left side of her face and ear as well as intermittent flashing lights and colors for an entire week. Roughly one week from the onset of the left hemifacial pain/headache, she had woken up to retro-orbital left eye pain and complained of double vision.

She saw her primary care physician (PCP) immediately, who prescribed promethazine and ketorolac, which provided minimal relief. She was started on a methylprednisone dose pack (4 mg tabs). C-reactive protein was checked and came back normal. Due to unresponsiveness to the treatments, she was referred to the emergency department (ED).

Her initial examination in the ED displayed a mild esotropia of the left eye in primary position and evidence of left lateral rectus palsy with subjective diplopia on left horizontal gaze. No other neurological deficits were noted. She was started on carbamazepine in the ED to treat for presumed trigeminal neuralgia, which subjectively helped her left hemifacial pain/headache
Correspondence: Ahmet Z. Burakgazi, Neuroscience Section, Department of Medicine, Virginia Tech Carilion School of Medicine, 3 Riverside Circle, Roanoke, VA 24016, USA.

Tel.: +1.540-521-4592

E-mail: drburakgazi@yahoo.com

Key words: Lyme disease, optic nerve, abducens nerve.

Contributions: the authors contributed equally.

Conflict of interest: the authors declare no potential conflict of interest.

Funding: none.

Received for publication: 15 July 2019.

Accepted for publication: 22 July 2019.

This work is licensed under a Creative Commons Attribution NonCommercial 4.0 License (CC BY-NC 4.0).

(C) Copyright: the Author(s), 2019

Licensee PAGEPress, Italy

Neurology International 2019; 11:8241

doi:10.4081/ni.2019.8241

mildly. She underwent clinical investigation with basic laboratory work, including comprehensive metabolic panel, blood cell count, and erythrocyte sedimentation rate, which all resulted in normal range values. She also underwent diagnostic imaging, including magnetic resonance venography (MRV) of head, which did not reveal cerebral venous sinus thrombosis, and magnetic resonance imaging (MRI) of brain with and without contrast, including thin cuts through the petrous ridge, which did not reveal any correlating pathology to her left lateral rectus palsy or headache.

Ophthalmology inpatient service was consulted, and examination revealed her visual acuity near with correction was 20/25 OD, 20/30 OS and tonometry was 17,16 $\mathrm{mm}$ hg respectively. Confrontation visual fields were grossly full bilaterally. There was no afferent pupil defect. Motility was full on the right eye. The left eye had an obvious esotropia in primary position, and she had clear signs of left abducens paresis. Funduscopic exam of the right eye was normal, while funduscopic exam of the left eye revealed a blurring of the neuro-retinal rim and optic nerve that was consistent with ON. Further tests, including Lyme titers with band and myasthenia panel, were ordered. Due to history of previous tick bite, she was placed on doxycycline $100 \mathrm{mg}$ twice a day, was discharged from the ED with an eye patch, and was ordered to follow up with her PCP, neurology, and neuro- 
ophthalmology for the results of her remaining lab work and further medical management.

Two days later, she developed a nonspecific scalp rash and immediately followed up with her PCP. She still expressed left facial pain and headache. She continued to have double vision from her left abducens palsy and visual blurriness in her left eye. Her laboratory test reviewed by her PCP resulted in borderline seropositive acetylcholine $(\mathrm{AcH})$ receptor binding antibodies of .56 (Positive: $>$ or $=0.50 \mathrm{nmol} / \mathrm{L}$ ). Her Lyme antibody screen was negative at $<0.90$ ( $>1.09$ is positive), but reflex IgG and IgM Western blot showed reactivity at $23 \mathrm{KD}$ IgG/IGM only. The decision was made to discontinue her doxycycline and to start her on valacyclovir 1 gr per day for seven days for the nonspecific rash. She was told to follow up with neurology for further management of her headache associated with ocular manifestations.

During her follow-up examination with neuro-ophthalmology on November 1, 2018, the patient's nonspecific rash had improved after two days of valacyclovir, and she also expressed subjective improvement of facial/head pain. The neuro-ophthalmology examination showed esotropia in primary position and limited abduction of the left eye, 20/20 visual acuities bilaterally, no relative afferent pupillary defect bilaterally, and normal color test bilaterally. Funduscopic exam revealed mild disc edema in the left eye with no further abnormalities seen, especially from the vessels, disc, or macula. Humphrey visual field analyzer in the left eye revealed an inferior nasal step defect. Given her left ipsilateral hemifacial pain, left sixth-nerve palsy, and unspecified mild left optic neuritis, the neuro-ophthalmologist was concerned about poly-cranial neuropathies and recommended a lumbar puncture to check for cell count and protein, cerebrospinal fluid (CSF) Lyme antibodies, CSF VDRL, oligoclonal bands, herpes simplex virus polymerase chain reaction (PCR), herpes zoster virus $\mathrm{PCR}$, and cytology. Pertinent results included mild pleocytosis of 22 cells (lymphocyte dominant), mild protein elevation of 49 , and normal serum glucose. The remainder of laboratory work ups including nonreactive CSF Lyme antibodies, negative CSF VDLR, and absent oligoclonal bands were within normal limits.

The patient followed up in the neurology clinic on December 3, 2018 and no longer expressed left hemifacial pain/headache but still had diplopia from the left abducens nerve palsy with double vision on examination. The patient also expressed a vague intermittent tingling sen- sation in bilateral feet, hands, and face, with a normal peripheral sensory nerve exam. She was put on pyridostigmine $60 \mathrm{mg}$ q4 to empirically treat for ocular myasthenia, given positive serology. Simultaneously, she was started once more on doxycycline $100 \mathrm{mg}$ bid to treat for LD, given her tick exposure, optic nerve involvement, and subjective complaints of paresthesias and dysgeusia. Myasthenia gravis panel serology was sent out from the December 3 neurology exam, which resulted on December 8 with negative $\mathrm{ACH}$ receptor binding, $\mathrm{ACH}$ receptor blocking and striated muscle antibodies.

The patient followed up with the neurology clinic on December 19, 2018, again without any further visual symptoms, and it was unclear if this improvement was a result of the pyridostigmine or doxycycline. The patient was then asked to discontinue her pyridostigmine and continue taking doxycycline to ensure that this was the result of tick-borne disease.

After a total of eight weeks on doxycycline, she remained asymptomatic and followed up on January 30, 2019 with an LD specialist, who repeated testing for Lyme antibody Western blot IgG/IgM. This time, she had a positive LD antibody IgM immunoblot reactive at $23 \mathrm{KD}, 39 \mathrm{KD}$, and $41 \mathrm{KD}$ but negative LD antibody $\operatorname{IgG}$ immunoblot, which was only reactive at 23KD and 41KD. The LD specialist concluded that the patient had LD and asked her to continue doxycycline for two more weeks.

The patient, having been on doxycycline for 10 weeks, followed up with neurology on February 11, 2019 without any subjective visual and sensory complaints. She was asked to continue doxycycline for two weeks longer.

\section{Discussion}

In this case report, we presented an atypical case of ipsilateral $\mathrm{ON}$ and sixthnerve palsy possibly related to LD. In our case, the patient initially presented with left ipsilateral hemifacial pain/headache, left sixth-nerve palsy, and papillitis. Due to the unusual presentation, she was started on pyridostigmine and doxycyline. The patient's visual symptoms and sensory complaints had resolved both subjectively and clinically while on both medications, and this improvement warranted repeat serology for MG, which was negative. The patient was stopped on pyridostigmine and continued on doxycycline with no further ocular and sensory manifestations. Repeated Lyme serology revealed a positive immunoblot of
IgM reactive at $23 \mathrm{KD}, 39 \mathrm{KD}$, and $41 \mathrm{KD}$, and immunoblot for $\mathrm{IgG}$ was reactive at $23 \mathrm{KD}$ and $41 \mathrm{KD}$. After 10 weeks of doxycycline, the patient continued to remain symptom free. To our best knowledge, this is first case report of LD related combined $\mathrm{ON}$ and abducens nerve palsy, which completely resolved with doxycycline treatment.

Laboratory confirmation of LD can be challenging. Typically, the $\mathrm{CDC}$ requires a two-step laboratory testing process for LD diagnosis, which includes enzyme-linked immunosorbent assay (ELISA) followed by a Western blot.21,22 The initial blood work up showed a negative Lyme AB ELISA screen followed by a Western blot that revealed only one identical $\operatorname{IgG} / \operatorname{IgM}$ band reactive at $23 \mathrm{KD}$. Repeat serology three months later resulted in positive IgM blot reactivity at $23 \mathrm{KD}, 39 \mathrm{KD}$, and $41 \mathrm{KD}$ and in positive five of the IgG blots. 1,23

Bands that are reactive on an IgM blot after four weeks can sometimes lead to false positive results, but it has been established that seroconversion from IgM to IgG can sometimes take up to two months. ${ }^{1}$ If one suspects neuroborreliosis, CSF for intrathecal production of antibodies to B. burgdorferi greater than 1.0 is considered a positive test, but it has been reported in case studies of early neuroborreliosis that CSF markers can be negative in $20-30 \%$ in the first six weeks.1,23 For this reason, it is paramount important to repeat LD tests in cases, which initial LD tests are negative but clinical suspicious is continuing.

Unfortunately, there is not a direct link between Lyme seropositivity and ON. A few studies have examined the relationship between LD and ON.16,23 Jacobson et al. ${ }^{23}$ performed an investigation of 20 patients with newly diagnosed isolated $\mathrm{ON}$ who resided in a region endemic for $\mathrm{LD}$, finding only four patients who had resulted in positive serology for LD. Three of these patients underwent CSF analysis, and two had CSF lymphocytic pleocytosis that remained unexplained after extensive evaluations for causes other than LD. ${ }^{15}$ Lyme disease related $\mathrm{CN} 6$ palsy is very rare. Few case reports $8,10,19,20$ in the literature have been reported. The simultaneous involvement of CN 3 and $\mathrm{CN} 6$ in a patient with LD was reported, 8 but the simultaneous involvement of $\mathrm{CN} 2$ and $\mathrm{CN} 6$ was not reported previously. In our case, the patient presented both $\mathrm{ON}$ and $\mathrm{CN} 6$ palsy.

Given the other neurological manifestations and $\mathrm{ON}$, it appears that these cases of $\mathrm{LD}$ in addition to $\mathrm{ON}$ are trending to a diagnosis of exclusion. Therefore, a comprehensive investigation was performed to rule out other causes of $\mathrm{ON}$ in addition to sixth- 
nerve palsy before beginning extensive treatment with doxycycline, which did show a favorable outcome. $3,13,15,20,24$

Neurological manifestations of LD in the past have shown highly effective responsiveness to ceftriaxone, cefotaxime, penicillin G, and doxycycline.4,14,15,24,25 Prior cases of patients who failed oral regimens showed response to IV ceftriaxone, high-dose penicillin, and cefotaxime. Studies in Europe ${ }^{26-30}$ have provided evidence that oral doxycycline can achieve effective concentrations in CNS and have been shown to be effective in Lyme meningitis, cranial neuritis, and radiculo-neuritis, as was the case in this patient.

\section{Conclusions}

Ocular manifestations of LD remain a rare feature of the disease, but it may present a wide range of clinical presentations with different combinations. The unique part of this case is that the patient presented with simultaneous involvement of optic and abducens nerves. The diagnosis of LD can be challenging and initial laboratory tests can be a false negative. It is paramount important to repeat the diagnostic test if clinical suspicious is ongoing. With this case, we aim to increase awareness of clinicians for possible ocular manifestations of LD and its complex diagnostic process.

\section{References}

1. Moussawi K, Gupta A, Reda H. Clinical Reasoning: A 20-year-old man with headache and double vision. Neurology 2016;87:e162-7.

2. Zrinscak O, Masnec-Paskvalin S, Corak $\mathrm{M}$, et al. Paralytic strabismus as a manifestation of lyme borreliosis. Coll Antropol 2005;29:137-9.

3. Balcer LJ, Winterkorn JM, Galetta SL. Neuro-ophthalmic manifestations of Lyme disease. J Neuroophthalmol 1997; 17:108-21.

4. Burakgazi AZ. Lyme disease-induced polyradiculopathy mimicking amyotrophic lateral sclerosis. Int J Neurosci 2014;124:859-62.

5. Halperin JJ, Logigian EL, Finkel MF, Pearl RA. Practice parameters for the diagnosis of patients with nervous system Lyme borreliosis (Lyme disease). Neurology 1996;46:619-27.

6. Halperin JJ, Volkman DJ, Wu P. Central nervous system abnormalities in Lyme neuroborreliosis. Neurology 1991;41: 1571-82.

7. Traisk F, Lindquist L. Optic nerve involvement in Lyme disease. Curr Opin Ophthalmol 2012; 23:485-90.

8. Lell M, Schmid A, Stemper B, et al. Simultaneous involvement of third and sixth cranial nerve in a patient with Lyme disease. Neuroradiology 2003;45:85-7.

9. Kauffmann DJ, Wormser GP. Ocular Lyme disease: case report and review of the literature. $\mathrm{Br} \mathrm{J}$ Ophthalmol 1990;74:325-7.

10. Lesser RL, Kornmehl EW, Pachner AR, et al. Neuro-ophthalmologic manifestations of Lyme disease. Ophthalmology 1990;97:699-706.

11. Wu G, Lincoff H, Ellsworth RM, Haik BG. Optic disc edema and Lyme disease. Ann Ophthalmol 1986;18:252-5.

12. Winterkorn JM. Lyme disease: neurologic and ophthalmic manifestations. Surv Ophthalmol 1990;35:191-204.

13. Lesser RL. Ocular manifestations of Lyme disease. Am J Med 1995;98:60S$2 \mathrm{~S}$.

14. Halperin JJ, Luft BJ, Anand AK, et al. Lyme neuroborreliosis: central nervous system manifestations. Neurology 1989;39:753-9.

15. Burakgazi AZ, Henderson CS. Unusual Presentation of Unilateral Isolated Probable Lyme Optic Neuritis. Case Rep Neurol Med 2016;2016:7471842.

16. Sibony P, Halperin J, Coyle PK, Patel K. Reactive Lyme serology in optic neuritis. J Neuroophthalmol 2005;25:71-82.

17. Park M. Ocular manifestations of Lyme disease. J Am Optom Assoc 1989;60:284-9.

18. Blanc F, Ballonzoli L, Marcel C, et al. Lyme optic neuritis. J Neurol Sci 2010;295:117-9.

19. Elder C, Hainline C, Galetta SL, et al. Isolated Abducens Nerve Palsy: Update on Evaluation and Diagnosis. Curr Neurol Neurosci Rep 2016;16:69.

20. Nair AG, Ambika S, Noronha VO, Gandhi RA. The diagnostic yield of neuroimaging in sixth nerve palsy-
Sankara Nethralaya Abducens Palsy Study (SNAPS): Report 1. Indian J Ophthalmol 2014;62:1008-12.

21. Engstrom SM, Shoop E, Johnson RC. Immunoblot interpretation criteria for serodiagnosis of early Lyme disease. J Clin Microbiol 1995;33:419-27.

22. Dressler F, Whalen JA, Reinhardt BN, Steere AC. Western blotting in the serodiagnosis of Lyme disease. J Infect Dis 1993;167:392-400.

23. Jacobson DM, Marx JJ, Dlesk A. Frequency and clinical significance of Lyme seropositivity in patients with isolated optic neuritis. Neurology 1991;41:706-11.

24. Halperin JJ, Shapiro ED, Logigian E, et al. Practice parameter: treatment of nervous system Lyme disease (an evidence-based review): report of the Quality Standards Subcommittee of the American Academy of Neurology. Neurology 2007;69:91-102.

25. Wormser GP, Dattwyler RJ, Shapiro $\mathrm{ED}$, et al. The clinical assessment, treatment, and prevention of lyme disease, human granulocytic anaplasmosis, and babesiosis: clinical practice guidelines by the Infectious Diseases Society of America. Clin Infect Dis 2006;43:1089134.

26. Lapp T. AAP issues recommendations on the prevention and treatment of Lyme disease. Am Fam Physician 2000;61:3463-4.

27. Pfister HW, Preac-Mursic V, Wilske B, Einhaupl KM. Cefotaxime vs penicillin $\mathrm{G}$ for acute neurologic manifestations in Lyme borreliosis. A prospective randomized study. Arch Neurol 1989;46:1190-4.

28. Bremell D, Dotevall L. Oral doxycycline for Lyme neuroborreliosis with symptoms of encephalitis, myelitis, vasculitis or intracranial hypertension. Eur J Neurol 2014;21:1162-7.

29. Ljostad U, Skogvoll E, Eikeland R, et al. Oral doxycycline versus intravenous ceftriaxone for European Lyme neuroborreliosis: a multicentre, non-inferiority, double-blind, randomised trial. Lancet Neurol 2008;7:690-5.

30. Mygland A, Ljostad U, Fingerle V, et al. EFNS guidelines on the diagnosis and management of European Lyme neuroborreliosis. Eur J Neurol 2010;17:816. 